\title{
SISTEMA DE INFORMAÇÃO EM SAÚDE: GESTÃO E ASSISTÊNCIA NO SISTEMA ÚNICO DE SAÚDE
}

Sérgio Ribeiro dos Santos', Jocelly de Araújo Fereira², Egillane Madza Mendes Santa Cruz ${ }^{3}$, Elanne Meire de Almeida Mendes Leite ${ }^{4}$, Juliana da Costa Santos Pessoa ${ }^{5}$

1Enfermeiro. Doutor em Sociologia. Universidade Federal da Paraíba. João Pessoa-PB-Brasil.

${ }^{2}$ Enfermeira. Mestre em Enfermagem. Universidade Federal de Campina Grande. João Pessoa-PB-Brasil.

${ }^{3}$ Enfermeira. Especialista em Gerenciamento de Serviços de Enfermagem. Secretaria de Saúde de João Pessoa. João Pessoa-PB-Brasil. ${ }^{4}$ Fisioterapeuta. Especialista em Fisioterapia Cardiorrespiratória. Home Care. João Pessoa-PB- Brasil.

${ }^{5}$ Fisioterapeuta. Doutoranda em Modelos de Decisão e Saúde. Centro Universitário de João Pessoa. João Pessoa-PB-Brasil.

RESUMO: O presente estudo objetiva investigar pesquisas realizadas sobre o sistema de informação em saúde utilizado pelo Sistema Único de Saúde e apontar as dificuldades de sua aplicabilidade. Trata-se de uma revisão integrativa efetuada por meio de uma busca bibliográfica online das produções científicas, no período de 2009 a 2013. Os artigos que compuseram o corpus de análise evidenciam a necessidade de desenvolver sistemas disponíveis para acesso e uso ao público, com ferramentas que possam oferecer dados consistentes que contribuam para otimizar a gestão assistencial prestada pelo Sistema Único de Saúde.

DESCRITORES: Sistemas de informação; Assistência à saúde; Sistema Único de Saúde.

\section{INFORMATION SYSTEM IN HEALTH: SISTEMA DE INFORMACIÓN EN SALUD: MANAGEMENT AND SUPPORT IN THE GESTIÓN Y ASISTENCIA EN EL SISTEMA ÚNICO BRAZILIAN UNIFIED HEALTH SYSTEM DE SALUD}

\begin{abstract}
This study aims to investigate studies on information system in health used by the Brazilian Unified Health System and to point out the difficulties in its applicability. This is an integrative review conducted through an online bibliographic search of the scientific production from 2009 to 2013. The articles that composed the corpus analysis revealed the need to develop systems for public access and usage by using tools able to offer consistent data and contribute to the optimization of support management provided by the National Unified Health System.
\end{abstract}

DESCRIPTORS: Information systems; Health care; Unified Health System (SUS).
RESUMEN: El presente estudio tiene la finalidad de analizar investigaciones realizadas sobre el sistema de información en salud utilizado por el Sistema Único de Salud y apuntar las dificultades de su aplicabilidad. Es una revisión integrativa efectuada por medio de una búsqueda bibliográfica online de las producciones científicas, en el periodo de 2009 a 2013. Los artículos que hicieron parte del corpus de análisis evidencían la necesidad de desarrollar sistemas disponibles para acceso y uso al público, con herramientas que ofrecen datos consistentes para contribuir para otimizar la gestión asistencial prestada por el Sistema Único de Salud.

DESCRIPTORES: Sistemas de información; Asistencia a la salud; Sistema Único de Salud. 


\section{INTRODUÇÃO}

O Sistema Único de Saúde (SUS) tem como objetivo principal garantir a saúde como direito do cidadão e dever do Estado, por meio de uma rede de ações e serviços regionalizada e hierarquizada. Para tanto, o SUS propõe o uso do conceito ampliado de saúde, que associa as condições de vida, norteia a formulação e a implementação de estratégias que viabilizem um serviço de saúde universal, integral, eficiente, com equidade e participação popular ${ }^{(1)}$.

Considerando que inúmeros fatores podem interferir no processo saúde-doença do indivíduo e que a demanda dos usuários tem aumentado, há a necessidade do uso de indicadores de assistência à saúde, com a finalidade de aferir o desempenho do sistema de serviços de saúde para nortear a gestão das políticas públicas e a tomada de decisões. Nessa perspectiva, a introdução das tecnologias de informação em saúde torna-se de fundamental importância ${ }^{(2)}$.

É necessário compreender que um sistema de informações envolve um conjunto de processos que se destina a capturar, processar, transformar, armazenar, manter e produzir informações ${ }^{(3)}$. Logo, um sistema de informação em saúde representa um meio para a obtenção de informações necessárias para que os serviços de saúde possam desenvolver e aplicar estratégias, que possibilitem melhores condições ao planejamento e à avaliação de ações para melhorias na área da saúde.

Tem-se observado, entretanto, que apesar do avanço no âmbito do sistema de informação em saúde com a finalidade de gerenciar informações e ações, ainda existem inúmeras dificuldades no seu desenvolvimento e na sua aplicabilidade, no que tange aos pré-requisitos fundamentais ao avanço do SUS. Essa aplicabilidade permite um aumento na capacidade de resposta do Estado e da sociedade, em prol da saúde da população brasileira, visto que envolve outros atores, práticas, procedimentos e saberes, que caracterizam a transdisciplinaridade em saúde ${ }^{(4)}$.

Nesse contexto, o presente estudo tem o objetivo de investigar a produção científica no tocante à temática "sistema de informação em saúde utilizado pelo SUS" e apontar dificuldades à sua aplicabilidade. Assim, este estudo buscará respostas aos seguintes questionamentos: de que forma os sistemas de informação no SUS têm sido aplicados? A aplicabilidade dos sistemas de informação de saúde tem contribuído, verdadeiramente, na amplitude e eficiência da gestão e da assistência aos usuários do SUS?

\section{MÉTODO}

Trata-se de um estudo bibliográfico documental realizado pelo método da revisão integrativa. Para a sua operacionalização, foram realizadas as seguintes etapas: determinação dos objetivos de estudo, formulação dos questionamentos a serem respondidos, identificação e coleta de pesquisas primárias relevantes. Após estas etapas, os estudos inicialmente coletados são avaliados criticamente, resultando consequentemente em uma redução do número de estudos incluídos na fase final da revisão, que serão analisados de maneira sistemática( ${ }^{(5)}$.

A pesquisa foi realizada por meio de busca online das produções científicas nacionais e internacionais, no período de 2009 a 2013, sobre Sistema de Informação em Saúde. O apoderamento dessas produções ocorreu por meio da Biblioteca Virtual em Saúde (BVS), sendo utilizadas as bases de dados: Literatura Latino-Americana e do Caribe em Ciências da Saúde (LILACS), Scientific Electronic Library Online (SciELO), Bases de Dados em Enfermagem (BDENF) e Medical Literature Analysis and Retrieval System Online (MEDLINE).

Os critérios para a seleção da amostra foram os seguintes: artigos disponíveis na íntegra; publicações dos últimos cinco anos; artigos editados nas línguas portuguesa e/ou inglesa. Os Descritores em Ciências da Saúde (DeCS) utilizados na busca foram: "Sistemas de Informação", "Sistema Único de Saúde" e "Assistência à Saúde".

Inicialmente, ao utilizar o descritor "Sistemas de Informação", obteve-se 72.072 artigos antes de serem filtrados com os critérios de inclusão, sendo 3.511 na base de dados LILACS e 62.926 na MEDLINE. Em relação ao descritor "Sistema Único de Saúde" foi encontrado 904 artigos, sendo 5.194 na base de dados LILACS, 221 na MEDLINE e 453 na BDENF. Em relação ao descritor "Assistência à Saúde", localizou-se 441.320, sendo 23.420 na base de dados LILACS, 386.431 na MEDLINE e 4.688 na BDENF.

Posteriormente, com a inclusão dos critérios acima descritos, encontrou-se o seguinte cenário: 418 artigos referentes a "Sistemas de Informação", sendo 100 na base de dados LILACS, 318 na MEDLINE e 453 na BDENF; "Sistema Único de Saúde" com 333 artigos, sendo 299 na base de 
dados LILACS e 34 na BDENF; por fim, "Assistência à Saúde" apresentando 5.093 artigos, sendo 4.725 na MEDLINE, 309 na LILACS e 59 na BDENF.

Ao confirmar o grande acervo de artigos com contextualizações diversas, que nem sempre abordavam em sua essência o conteúdo proposto por este estudo, acreditou-se ser mais relevante o cruzamento dos três descritores, utilizando dessa forma a seguinte proposição: "Sistemas de Informação AND Sistema Único de Saúde AND Assistência à Saúde", sendo estes três descritores utilizados simultaneamente em uma mesma pesquisa. Diante desse cruzamento foram apreendidos 19 artigos, sendo 9 na base de dados LILACS e 10 na SciELO.

Notou-se que dos 10 artigos presentes na SciELO, três deles eram iguais aos artigos encontrados na LILACS. Excluindo as repetições, contabilizouse apenas uma vez. Portanto, dos 19 artigos encontrados, 16 foram incluídos na pesquisa, pois estavam relacionados à temática, ao objetivo desse estudo e contemplavam os critérios de inclusão estabelecidos.

Os estudos selecionados foram analisados de maneira crítica e detalhada, procurando explicações para os resultados diferentes ou conflitantes. Para tanto, visando facilitar a visualização estrutural e lógica deste estudo, fez-se a confecção de fichas de leitura, com o intuito de destacar as unidades de registro para agrupar os diferentes temas e, posteriormente, proceder a discussão do estudo, com os principais resultados evidenciados mediante análise dos artigos ora incluídos ${ }^{(5)}$.

\section{RESULTADOS}

Os artigos foram analisados e descritos, em síntese, através dos Quadros 1 e 2, abordando o ano de publicação, a base de dados, a língua de publicação, o periódico, o tipo de estudo, os sistemas de informação abordados e a sua utilização.

Os resultados expressos nos Quadros 1 e 2, bem como na discussão abaixo descrita, mostraram que os sistemas de informação em saúde trazem em seu escopo a magnitude de auxiliar a gestão municipal, estadual e federal em relação aos programas necessários à administração e manutenção da saúde. Torna-se imperioso ressaltar que em relação à assistência, os sistemas de informação ainda necessitam de um aprimoramento, a fim de melhor atender às necessidades iminentes dos usuários e dos profissionais de saúde.

Perante esses resultados, faz-se necessário discutir mais detalhadamente as afirmativas expressas.

Quadro 1 - Descrição dos artigos selecionados de 2009 a 2013, segundo o ano de publicação, base de dados, língua de publicação, nome do periódico e tipo de estudo. João Pessoa-PB-Brasil, 2013

\begin{tabular}{|l|l|l|l|l|l|}
\hline Artigo & Ano & Bases de dados & Lingua de publicação & \multicolumn{1}{|c|}{ Nome do periódico } & Tipo de estudo \\
\hline Artigo 1 & 2009 & Scielo & Português & Revista Brasileira de Saúde Materno Infantil & Exploratório \\
\hline Artigo 2 & 2009 & Scielo & Português & Texto Contexto Enfermagem & Caso \\
\hline Artigo 3 & 2009 & Lilacs & Português & Revista Médica de Minas Gerais & Ecológico \\
\hline Artigo 4 & 2010 & Scielo & Português & Caderno de Saúde Pública & Exploratório \\
\hline Artigo 5 & 2010 & Scielo & Português/Inglês & Revista de Saúde Pública & Transversal \\
\hline Artigo 6 & 2011 & Scielo & Português/Inglês & Caderno de Saúde Pública & Ecológico \\
\hline Artigo 7 & 2011 & Lilacs & Português & Revista Baiana de Saúde Pública & Exploratório \\
\hline Artigo 8 & 2011 & Scielo & Português & Revista Brasileira de Epidemiologia & Exploratório \\
\hline Artigo 9 & 2011 & Lilacs & Português & Scientia Médica & Revisão \\
\hline Artigo 10 & 2011 & Scielo & Português/Inglês & Revista de Saúde Pública & Quantitativo \\
\hline Artigo 11 & 2011 & Scielo & Português/Inglês & Revista de Saúde Pública & Transversal \\
\hline Artigo 12 & 2012 & Scielo & Português/Inglês & Revista de Saúde Pública & $\begin{array}{l}\text { Ecológico/ } \\
\text { Retrospectivo }\end{array}$ \\
\hline Artigo 13 & 2012 & Scielo & Português & Revista Brasileira de Epidemiologia & $\begin{array}{l}\text { Descritivo/ } \\
\text { Exploratório }\end{array}$ \\
\hline Artigo 14 & 2012 & Lilacs & Português & Revista Brasileira de Reumatologia & Transversal \\
\hline Artigo 15 & 2012 & Scielo & Português/Inglês & Revista de Saúde Pública & Ecológico \\
\hline Artigo 16 & 2012 & Scielo & Português/Inglês & Revista de Saúde Pública & $\begin{array}{l}\text { Descritivo/ } \\
\text { Exploratório }\end{array}$ \\
\hline
\end{tabular}


Quadro 2 - Descrição dos artigos selecionados, segundo o sistema de informação referenciado e a finalidade da utilização do sistema de informação. João Pessoa-PB-Brasil, 2013

\begin{tabular}{|c|c|c|}
\hline Artigo & Sistema de informação abordado & Utilização do sistema de informação \\
\hline Artigo 1 & $\begin{array}{l}\text { Sistema de Informação Hospitalar do Sistema Único de Saúde } \\
\text { (SIH/SUS) }\end{array}$ & Fonte de dados \\
\hline Artigo 2 & $\begin{array}{l}\text { Sistema de Informação da Atenção Básica (SIAB) e Sistema de } \\
\text { Cadastro Nacional de Portadores de Hipertensão e Diabetes (SIS/ } \\
\text { HIPERDIA) }\end{array}$ & Estudo de caso \\
\hline Artigo 3 & $\begin{array}{l}\text { Sistema de Informação de Mortalidade (SIM) e Sistema de } \\
\text { Informação de Nascidos Vivos (SINASC) }\end{array}$ & Fonte de dados \\
\hline Artigo 4 & $\begin{array}{l}\text { Autorização de Procedimento de Alta Complexidade do Sistema } \\
\text { de Informações Ambulatoriais do Sistema Único de Saúde } \\
\text { (APACSIA/SUS/ONCO) }\end{array}$ & $\begin{array}{l}\text { Fonte de dados } \\
\text { Desenvolvimento de um SI }\end{array}$ \\
\hline Artigo 5 & $\begin{array}{l}\begin{array}{l}\text { Sistema de Informação Hospitalar do Sistema Único de Saúde } \\
\text { (SIH/SUS) }\end{array} \\
\end{array}$ & Fonte de dados \\
\hline Artigo 6 & $\begin{array}{l}\text { Sistema de Informação Hospitalar do Sistema Único de Saúde } \\
\text { (SIH/SUS) e Autorização de Procedimento de Alta Complexidade } \\
\text { do Sistema de Informações Ambulatoriais do Sistema Único de } \\
\text { Saúde (APACSIA/SUS/ONCO) }\end{array}$ & Fonte de dados \\
\hline Artigo 7 & $\begin{array}{l}\text { Sistema de Informação Hospitalar do Sistema Único de Saúde } \\
\text { (SIH/SUS) e o Cadastro Nacional de Estabelecimentos de Saúde } \\
\text { (CNES) }\end{array}$ & Fonte de dados \\
\hline Artigo 8 & $\begin{array}{l}\text { Cadastro Nacional de Estabelecimentos de Saúde (CNES); } \\
\text { Sistema de Informação Hospitalar do Sistema Único de Saúde } \\
\text { (SIH/SUS); e Comunicação de Internações Hospitalares (CIH) }\end{array}$ & Fonte de dados \\
\hline Artigo 9 & Prontuário Eletrônico do Paciente (PEP) & Avaliação do sistema de informação \\
\hline Artigo 10 & $\begin{array}{l}\text { Sistema de Informações sobre Orçamentos Públicos em Saúde } \\
\text { (SIOPS); e Siga Brasil }\end{array}$ & Fonte de dados \\
\hline Artigo 11 & \begin{tabular}{|l|} 
Sistema de Acompanhamento do Programa de Humanização no \\
Pré-Natal e Nascimento (SISPRENATAL)
\end{tabular} & Fonte de dados \\
\hline Artigo 12 & $\begin{array}{l}\begin{array}{l}\text { Sistema de Informação Hospitalar do Sistema Único de Saúde } \\
\text { (SIH/SUS); e Sistema de Informação da Atenção Básica (SIAB) }\end{array} \\
\end{array}$ & Fonte de dados \\
\hline Artigo 13 & $\begin{array}{l}\text { Sistema de Informação Hospitalar do Sistema Único de Saúde } \\
\text { (SIH/SUS) }\end{array}$ & Fonte de dados \\
\hline Artigo 14 & \begin{tabular}{|l} 
Sistema de Informação do Componente Especializado da \\
Assistência Farmacêutica (HÓRUS Especializado)
\end{tabular} & Fonte de dados \\
\hline Artigo 15 & $\begin{array}{l}\text { Sistema de Informação Hospitalar do Sistema Único de Saúde } \\
\text { (SIH/SUS) }\end{array}$ & Fonte de dados \\
\hline Artigo 16 & $\begin{array}{l}\text { Sistema Nacional de Gestão da Assistência Farmacêutica } \\
\text { (HÓRUS) }\end{array}$ & Avaliação do sistema de informação \\
\hline
\end{tabular}

\section{DISCUSSÃO}

Os vários Sistemas de Informação em Saúde (SIS), sejam eles assistenciais ou epidemiológicos implantados pelo Ministério da Saúde (MS) nas últimas décadas, têm sido referenciados como ferramentas importantes para o diagnóstico de situações de saúde, com o propósito de gerar intervenções mais condizentes com as necessidades da população.

Nessa pesquisa, ao ler os artigos, pode-se verificar a preocupação dos autores em utilizar os
SIS como fonte de dados para ações de controle, monitoramento e avaliação da atenção à saúde no Brasil.

Dos estudos encontrados, oito desenvolveram pesquisas referentes à análise e avaliação dos serviços hospitalares públicos e privados conveniados ao SUS, baseado nos dados ofertados pelo SIH-SUS, que é um instrumento utilizado em maior escala para a apreensão dos dados necessários ao desenvolvimento de pesquisas $^{(6-7)}$.

Um dos artigos pesquisados, o SIH-SUS foi 
analisado para caracterizar aspectos da assistência hospitalar aos desnutridos menores de cinco anos. Nesse estudo, verificou-se a necessidade dos profissionais de saúde valorizar a avaliação do estado nutricional e seu correto registro nos $\mathrm{SIH}$ SUS ${ }^{(8)}$. Em outro estudo, foi possível desenvolver um modelo preditivo de óbito hospitalar com base nos dados do SIH-SUS, confirmando dessa forma que quando devidamente registrado, há confiabilidade dos dados presentes nesse sistema para a avaliação do desempenho hospitalar(9).

Os dados do SIH-SUS também foram usados para avaliar a utilização de exames de tomografia computadorizada em internações e diagnóstico de Acidente Vascular Cerebral no SUS. Contudo, observou-se que será necessária a realização de outros estudos para saber se a realização desse exame trouxe melhoria no cuidado prestado e, consequentemente, compreender em que medida os resultados obtidos seriam produto da qualidade da informação no SIH-SUS ${ }^{(10)}$.

Percebe-se, então, que os estudos desenvolvidos e analisados ainda enfatizam as doenças, portanto há necessidade de mais pesquisas que detalhem o SIH-SUS e avaliem a qualidade dos dados emitidos por tal sistema.

Essa situação foi evidenciada no estudo "O Sistema de Informação Hospitalar e sua aplicação na saúde coletiva", em que levantou a produção científica envolvendo aplicações dos dados do SIH/SUS na Saúde Coletiva, com o propósito de sintetizar as vantagens e limitações desse sistema. Nesse estudo, foi possível verificar a cobertura incompleta das internações hospitalares pelo $\mathrm{SIH} / \mathrm{SUS}$ e incertezas quanto à confiabilidade das informações contidas no SIH/SUS, de forma que pode ser controvertida sua contribuição na pesquisa, organização e avaliação da assistência médico-hospitalar ${ }^{(11)}$.

Os dados obtidos pelo SIH-SUS nem sempre subsidiam informações necessárias à totalidade de uma pesquisa, dessa forma, precisa-se da introdução de outros sistemas para complementar os dados importantes aos estudos. Entre esses sistemas, pode-se citar: o módulo de oncologia do subsistema da Autorização de Procedimento de Alta Complexidade do Sistema de Informações Ambulatoriais do Sistema Único de Saúde (APACSIA/SUS/ONCO), o Cadastro Nacional de Estabelecimentos de Saúde (CNES), a Comunicação de Internações Hospitalares $(\mathrm{ClH})$ e o Sistema de Informação da Atenção Básica $(\mathrm{SIAB})^{(12)}$. Sendo assim, torna-se indispensável que os dados fornecidos aos sistemas sejam devidamente alimentados e processados para um eficaz planejamento e distribuição dos serviços.

Utilizou-se o APACSIA/SUS/ONCO em conjunto com o SIH-SUS, por ser o sistema utilizado para gerir as terapêuticas e os recursos destinados aos tratamentos dos pacientes oncológicos do SUS, além de dimensionar a infraestrutura assistencial e melhorar a gestão da assistência oncológica ambulatorial, através do SISONCO, sistema esse desenvolvido em um dos estudos da amostra da pesquisa ${ }^{(1,12)}$.

Além dessas finalidades, o SIH-SUS junto ao CNES em um dos artigos, também foi utilizado como fonte de dados para discutir a situação atual e as tendências da atenção hospitalar do SUS. Essa característica dos sistemas é importante para demonstrar que há uma tendência à ampliação de unidades públicas de maior porte, com maior adensamento tecnológico, sem evidência de ações voltadas para uma reorientação do perfil dos hospitais de pequeno porte ${ }^{(13)}$.

Em outro artigo contemplado nesse estudo, os autores utilizaram o CNES e o SIH-SUS associados à Comunicação de Internações Hospitalares $(\mathrm{ClH})$, para o alcance dos objetivos de sua pesquisa. Quando utilizado pela Agência Nacional de Saúde Suplementar, o $\mathrm{CIH}$ avalia a rede assistencial informada pelos planos privados de assistência à saúde, permitindo, assim, um conhecimento mais amplo e profundo dos perfis nosológico e epidemiológico da população brasileira $^{(14)}$.

O estudo "Hospitalizações por condições cardiovasculares sensíveis à atenção primária em municípios goianos", contemplado nesta revisão, usou o SIAB interligado ao SIH-SUS para avaliar as taxas de hospitalizações por condições cardiovasculares sensíveis à atenção primária. Observou-se, nesse estudo, que as taxas de hospitalizações diminuíram independentemente da cobertura da Saúde da Família ${ }^{(15)}$.

O SIAB, utilizado em outra pesquisa aliado ao Sistema de Cadastro Nacional de Portadores de Hipertensão e Diabetes (SIS/HIPERDIA), realizou a análise situacional em saúde e/ou produção de informações para o planejamento da assistência e a resolutividade das ações de saúde ${ }^{(16)}$. 
Assim, diante da contextualização desta revisão, até o momento enfatizou-se como o SIH-SUS tem contribuído para o desenvolvimento das pesquisas científicas. A partir de então, abordar-se-ão outros sistemas tão relevantes quanto o SIH-SUS.

O Sistema de Informação de Mortalidade (SIM) e o Sistema de Informação de Nascidos Vivos (SINASC) foram abordados em um artigo que objetivou avaliar e analisar criticamente a qualidade da informação em saúde e o papel de instituições e profissionais de saúde sobre esse tema ${ }^{(17)}$. Os autores observaram que o preenchimento da declaração de óbito continua apresentando vários problemas, tais como a falta de registro em campos obrigatórios, o que dificulta o correto diagnóstico situacional, mais uma vez atestando a necessidade de investimentos para o aperfeiçoamento dos sistemas e dos alimentadores, a fim de desenvolver fonte geradora de dados fundamentais para o planejamento e avaliação de políticas públicas de saúde.

A falta de registro, também, foi um problema observado no Sistema de Acompanhamento do Programa de Humanização no Pré-Natal e Nascimento (SISPRENATAL) ${ }^{(18)}$. Os sistemas de informação utilizados para discussão sobre financiamentoe planejamento doSUS também foram contemplados na amostra desse estudo, como é o caso do Sistema de Informações sobre Orçamentos Públicos em Saúde (SIOPS) e do Siga Brasil ${ }^{(19-20)}$.

Diante do exposto, identificou-se que a grande maioria dos sistemas de informação tratados, até o momento, foi empregada como fonte de dados para o desenvolvimento dos estudos abordados. Algumas pesquisas, entretanto, também realizaram uma avaliação de determinados sistemas de informação com a finalidade de demonstrar sua importância e as contribuições/dificuldades que os mesmos oferecem durante sua utilização.

Evidenciou-se tal situação nos artigos que abordaram o Sistema Nacional de Gestão da Assistência Farmacêutica (HÓRUS) e o Prontuário Eletrônico do Paciente (PEP), em que os resultados de sua aplicabilidade foram analisados, constatando-se que apesar das dificuldades existentes, a utilização dos mesmos ainda é válida por contribuir na melhoria das condições de saúde ${ }^{(2,21-22)}$.

Percebe-se ao longo da análise dos artigos selecionados que os sistemas de informação utilizados pelo SUS são instrumentos importantes e necessários para a obtenção de dados, construção da informação e utilização da mesma para o desenvolvimento de ações direcionadas ao controle, acompanhamento e avaliação da saúde no território nacional.

Alguns desses sistemas de informação, ainda assim, enfrentam problemas, pois não existe uma política que incentive o registro correto dos dados, o que concorre entre outros aspectos para o descaso no momento de codificar, impedindo (muitas vezes) de gerar informações corretas e, consequentemente, desenvolver ações eficazes, sendo visível a necessidade de adequação desses sistemas à nossa situação de saúde. Agrega-se a isso, a carência de um treinamento sistemático dos profissionais responsáveis por essas atividades.

\section{CONCLUSÃO}

Pode-se perceber que apesar da relevância que o tema possui para a saúde, ainda apresenta fragilidades na sua forma de organização e prática, o que compromete a confiabilidade dos dados produzidos, não permitindo que os sistemas representem a realidade da situação de saúde da população brasileira.

O presente estudo também revelou que os artigos apreendidos e discutidos, em sua grande maioria, não trazem os Sistemas de Informação como assunto principal. Eles utilizam os sistemas como ferramenta para concretude dos dados necessários ao desenvolvimento de pesquisa, externando os sistemas de informação em saúde nas metodologias como instrumentos de onde capturaram os dados de assuntos diversos na saúde.

Outro fator relevante é que os artigos trouxeram em seu escopo que o SIS disponibilizado pelo SUS é bem mais utilizado para a captura de dados administrativos, para custos financeiros, no auxílio de decisões gerenciais, quando comparado a sua utilização na assistência direta ao usuário do SUS. Contudo, ainda percebeu-se com este estudo, o quanto o SI visibiliza a gestão e invisibiliza o usuário, realidade que pode ser mudada com a existência dos prontuários eletrônicos.

Diante do exposto, surgem as seguintes reflexões: por que os profissionais da saúde não desenvolvem os SIS necessários à assistência oferecida por eles? Por que a alimentação dos SIS do SUS é realizada por técnicos, ao invés de profissionais, que são quem produzem os dados 
e quem conhecem a realidade do usuário?

A partir desses questionamentos, percebe-se a necessidade de capacitação dos profissionais da saúde que estão diretamente assistindo ao usuário do SUS e que produzem os dados alimentadores dos sistemas, a partir de suas consultas e atividades, além do desenvolvimento de sistemas que sejam livres, disponíveis ao público para acesso e uso, com ferramentas que possam contribuir para melhoria da gestão assistencial prestada pelo SUS.

\section{REFERÊNCIAS}

1. Gomes Junior SCS, Almeida RT. Modelo de simulação para estimar a infraestrutura necessária à assistência oncológica no sistema público de saúde. Rev. panam. salud publica. [Internet] 2009;25(2) [acesso em 15 out 2013]. Disponível: http://dx.doi.org/10.1590/S1020-49892009000200003

2. Costa KS, Nascimento Junior JM. HÓRUS: Inovação tecnológica na assistência farmacêutica no sistema único de saúde. Rev. Saude Publica. [Internet] 2012;46(1) [acesso em 15 out 2013]. Disponível: http:// dx.doi.org/10.1590/S0034-89102012005000063

3. Scherer V, Miranda FMD, Sarquis LMM, Lacerda MR. Sinan net: um sistema de informação à vigilância na saúde do trabalhador. Cogitare enferm. [Internet] 2007;12(3). [acesso em 21 out 2013]. Disponível: http://ojs.c3sl.ufpr.br/ojs2/index.php/cogitare/article/ view/10027/6888

4. Moraes IHS, Gomez MNG. Informação e informática em saúde: caleidoscópio contemporâneo da saúde. Ciênc. saúde colet. [Internet] 2007;12(3) [acesso em 15 out 2013]. Disponível: http://dx.doi.org/10.1590/ S1413-81232007000300002

5. Mendes KDS, Silveira RCCP, Galvão CM. Revisão integrativa: método de pesquisa para a incorporação de evidências na saúde e na enfermagem. Texto Contexto Enferm. [Internet] 2008;17(4) [acesso em 05 ago 2014]. Disponível: http://dx.doi.org/10.1590/ S0104-07072008000400018

6. Lemos C, Chaves LDP. Produção de internações hospitalares, no sistema único de saúde, na região de Ribeirão Preto, Brasil. Rev. Gaúcha Enferm. [Internet] 2011;32(4) [acesso em 25 out 2013]. Disponível: http:// dx.doi.org/10.1590/S1983-14472011000400013

7. Lobato G, Reichenheim ME, Coeli CM. Sistema de informações hospitalares do sistema único de saúde (SIH-SUS): uma avaliação preliminar do seu desempenho no monitoramento da doença hemolítica perinatal Rh(D). Cad. Saúde Pública. [Internet] 2008;24(3) [acesso em 25 out 2013]. Disponível: http://
dx.doi.org/10.1590/S0102-311X2008000300014

8. Bittencourt SA, Niquini RP, Reis AC, Leal MC. Assistência a crianças desnutridas: análise de dados do sistema de informação hospitalar do sistema único de saúde do Brasil. Rev. bras. saude mater. infant. [Internet] 2009;9(3) [acesso em 15 out 2013]. Disponível: http:// dx.doi.org/10.1590/S1519-38292009000300005

9. Gomes AS, Klück MM, Riboldi J, Fachel JMG. Modelo preditivo de óbito a partir de dados do sistema de informações hospitalares. Rev. Saude Publica. [Internet] 2010;44(5) [acesso em 15 out 2013]. Disponível: http:// dx.doi.org/10.1590/S0034-89102010005000037

10. Rolim CLRC, Martins M. O uso da tomografia computadorizada nas internações por acidente vascular cerebral no sistema único de saúde no Brasil. Rev. Bras. Epidemiol. [Internet] 2012;15 (1) [acesso em 15 out 2013]. Disponível: http://dx.doi.org/10.1590/ S1415-790X2012000100016

11. Bittencourt SA, Camacho LAB, Leal MC. O sistema de informação hospitalar e sua aplicação na saúde coletiva. Cad. Saúde Pública. [Internet] 2006;22(1) [acesso em 25 out 2013]. Disponível: http://dx.doi. org/10.1590/S0102-311X2006000100003

12. Oliveira EXG, Melo ECP, Pinheiro RS, Noronha $\mathrm{CP}$, Carvalho MS. Acesso à assistência oncológica: mapeamento dos fluxos origem-destino das internações e dos atendimentos ambulatoriais. O caso do câncer de mama. Cad. Saúde Pública. [Internet] 2011;27(2) [acesso em 15 out 2013]. Disponível: http://dx.doi. org/10.1590/S0102-311X2011000200013

13. Souza RC, Freire SM, Almeida RT. Sistema de informação para integrar os dados da assistência oncológica ambulatorial do sistema único de saúde. Cad. Saúde Pública. [Internet] 2006;26(6) [acesso em 15 out 2013]. Disponível: http://dx.doi.org/10.1590/ S0102-311X2010000600007

14. Barretto LD, Freire RC. Tendências da atenção hospitalar no SUS da Bahia: uma análise de 2006 a 2009. Rev. baiana de saude publica. [Internet] 2011;35(2) [acesso em 15 out 2013]. Disponível: http://inseer.ibict.br/rbsp/index.php/rbsp/article/ viewFile/309/pdf_118

15. Ministério da Saúde (BR). DATASUS [Internet]. Brasília: DF, 2008 [acesso em 20 out 2013]. Disponível: http:// www2.datasus.gov.br/DATASUS/index.php

16. Batista SRR, Jardim PCBV, Sousa ALL, Salgado CM. Hospitalizações por condições cardiovasculares sensíveis à atenção primária em municípios goianos. Rev. Saude Publica. [Internet] 2012;46(1) [acesso em 15 out 2013]. Disponível: http://dx.doi.org/10.1590/ S0034-89102012005000001

17. Thaines GHLS, Bellato R, Faria APS, Araújo LFS. 
Produção, fluxo e análise de dados do sistema de informação em saúde: um caso exemplar. Texto Contexto Enferm. [Internet] 2009;18(3) [acesso em 15 out 2013]. Disponível: http://dx.doi.org/10.1590/ S0104-07072009000300009

18. Graciano MMC, Araújo EW, Nogueira DA. Sistema de informação em saúde e atuação do profissional médico. Rev. Med. Minas Gerais. 2009;19(3):198-205.

19. Andreucci CB, Cecatti JG, Macchetti CE, Sousa $\mathrm{MH}$. Sisprenatal como instrumento de avaliação da qualidade da assistência à gestante. Rev. Saude Publica. [Internet] 2011;45(5) [acesso em 15 out 2013]. Disponível: http://dx.doi.org/10.1590/S003489102011005000064

20. Ministério da Saúde (BR). DATASUS. SIOPS - Sistema de Informações sobre Orçamentos Públicos em Saúde [Internet]. Brasília, DF: 2010 [acesso em 22 out 2013] Disponível: http://bvsms.saude.gov.br/bvs/folder/ sistema_informacao_orcamentos_publicos_saude.pdf

21. Vieira FS, Zucchi P. Aplicações diretas para aquisição de medicamentos no sistema único de saúde. Rev. Saude Publica. [Internet] 2011;45(5) [acesso em 15 out 2013] Disponível: http://dx.doi.org/10.1590/S003489102011005000048

22. Patrício CM, Maia MM, Machiavelli JL, Navaes MA. O prontuário eletrônico do paciente no sistema de saúde brasileiro: uma realidade para os médicos? Sci. Med. [Internet] 2011;21(3) [acesso em 15 out 2013]. Disponível: http://revistaseletronicas.pucrs.br/ojs/ index.php/scientiamedica/article/view/8723/6722php/ scientiamedica/article/view/8723/6722 